\title{
Prevalencia del estado periodontal de pacientes portadores de prótesis parcial removible tratados en una clínica dental privada.
}

Prevalence of periodontal status of patients with removable partial dentures treated in a private dental clinic.

Gian Marco Gonzáles ${ }^{1, a}$, Beatriz Chávez-Reategui ${ }^{1, c}$, Jorge Manrique-Chávez ${ }^{1, b}$, Ivonne Hidalgo-López ${ }^{1, b}$

\section{RESUMEN}

Objetivo: Identificar la prevalencia del estado periodontal de los pacientes portadores de prótesis parcial removible que acudieron a la Clínica Dental Docente de la UPCH. Materiales y métodos: Diseño retrospectivo con universo conformado por pacientes portadores de prótesis parcial removible recepcionados por alumnos de pregrado. Resultados: Se incluyeron a 130 historias clínicas encontrando mayor prevalencia del género femenino (66.92\%), con una edad media de 43.57 años. En el análisis univariado se encontró mayor prevalencia en la arcada inferior (61.54\%), clase III de edentulismo (57.69\%), estado gingival leve (55.38\%), higiene oral deficiente (46.92\%), ausencia de movilidad (91.54\%), presencia de retención (88.46\%), ausencia de recesión (79.23\%), defecto de furcación (100\%), estado malo de la PPR (73.85\%) y estado periodontal enfermo (96.92\%). En el análisis bivariado, se encontró relación entre las variables estado periodontal - clase de edentulismo y estado gingival - higiene oral; por otro lado no se encontró relación entre estado gingival - clase de edentulismo, estado de prótesis - estado gingival, estado periodontal - género, estado periodontal - arcada dentaria, recesión gingival - retención de placa, estado de prótesis - movilidad dentaria y estado periodontal - estado de prótesis. Conclusiones: Es de vital importancia realizar controles rigurosos del estado periodontal antes, durante y después de cualquier tratamiento realizado en la cavidad bucal, para garantizar su funcionalidad, longevidad y por ende bienestar al paciente.

\section{Palabras clave: PREVALENCIA, PRÓTESIS DENTAL, PERIODONCIA, OCLUSIÓN DENTAL.}

\section{ABSTRACT \\ ${ }^{1}$ Facultad de Estomatología Roberto Beltrán. Universidad Peruana Cayetano Heredia. Lima, Perú. \\ a Bachiller en Estomatología. \\ b Maestría en Estomatología.}

Objective: To identify the prevalence of periodontal status in patients fitted with removable partial dentures attending at the Teaching Dental Clinic (UPCH). Materials and methods: A retrospective design was conducted with a universe of patients fitted with removable partial dentures seen by undergraduate students. Results: A total of 130 dental records were analyzed, of those $66.92 \%$ were females with a mean age of 43.57 years old. The 
Univariate analysis found a higher prevalence in the lower arch (61.54\%), Class III of edentulism (57.69\%), mild gingival status (55.38\%), poor oral hygiene (46.92\%), lack of mobility (91.54\%), presence of retention $(88.46 \%)$, absence of recession (79.23\%), furcation defect (100\%), poor condition of RPD (73.85\%) and periodontal disease $(96.92 \%)$. In the Bivariate analysis, the related variables were: periodontal status with Class of edentulism and gingival status with oral hygiene; the unrelated variables were: gingival status with Class of edentulism, prosthetic status with gingival status, periodontal status with gender, periodontal status with dental arch, gingival recession with plaque retention, denture status with tooth mobility and periodontal status with prosthesis status. Conclusions: It is of vital importance perform a rigorous evaluation of the periodontal status before, during and after any treatment performed in the oral cavity, to ensure their functionality, longevity and therefore patient welfare.

\section{Key words: PREVALENCE, DENTURES, PERIODONTICS, DENTAL OCCLUSION.}

\section{INTRODUCCIÓN}

Para la correcta evaluación periodontal en pacientes portadores de prótesis parcial removibles (PPR) es indispensable aplicar y analizar una serie de índices que nos permitan identificar eficazmente el estado actual del periodonto. De esta manera, el profesional puede calificar el estado periodontal de las piezas comprometidas con la PPR.

El periodonto es un sistema funcional que comprende la encía, ligamento periodontal, el cemento radicular y hueso alveolar, la función principal consiste en transmitir y amortiguar las fuerzas masticatorias, y su capacidad propioceptora ayuda a mantener la integridad en la superficie de la mucosa masticatoria de la cavidad bucal. Constituye una unidad de desarrollo biológico y funcional que sufre cambios atribuibles a factores como la edad, adicción, alteraciones oclusales y medio bucal (1). Los índices más utilizados son (2,3): Índice Gingival (Loe y Silness, 1963), índice de Retención (Bjorby y Loe, 1967), índice de Movilidad Dentaria (Miller, 1938), índice de Higiene Oral Simplificado (Green y Vermillion, 1964), índice de Recesiones Gingivales (Miller, 1985) e índice de Furcación (Hamp et al. 1975)

El edéntulo parcial logra restablecer la función masticatoria, fonética, deglutiva y en algunos casos estética a través de la confección de la PPR. Por la gran variedad de casos encontrados de edentulismo parcial, Edward Kennedy en 1925 vio la necesidad de establecer una clasificación, que permite tener una idea más cercana del estado de conservación de los dientes y realizar de manera más efectiva el diseño de la PPR (4). Muchas veces las PPR al actuar mecánicamente sobre los tejidos, dan lugar a cambios de diversas índoles que están en relación con la capacidad de los tejidos y la forma de agresión que ejerza la prótesis $(5,6)$.

Los hallazgos en la literatura con respecto a la PPR y sus repercusiones periodontales presentan resultados desfavorables. En nuestro medio existen pocos trabajos de investigación acerca de este tema, por tal motivo se plantea este estudio para estar al tanto de las necesidades de atención de dicha población.

El objetivo el estudio fue identificar la prevalencia del estado periodontal de los pacientes portadores de prótesis parcial removible (PPR) que acudieron a la Clínica Dental Docente de la UPCH en los años 2011 y 2012 .

\section{MATERIALES Y MÉTODOS}

Diseño de tipo retrospectivo, observacional, descriptiva y de corte transversal que estudio la prevalencia del estado periodontal de los pacientes portadores de PPR que acudieron a la Clínica Dental de la UPCH en los años 2011 y 2012. El tamaño del universo estudiado estuvo conformado por 130 historias clínicas para la elaboración de esta investigación.

Los criterios de inclusión fueron: pacientes recepcionados por los alumnos de pregrado de la Facultad de Estomatología de la UPCH, pacientes entre los 18 a 64 años y pacientes portadores de prótesis parcial removible (PPR) y los criterios de exclusión fueron: pacientes con tratamiento 
ortodóncicos, pacientes con tratamientos médicos (últimos 6 meses), pacientes con tratamientos quirúrgicos, pacientes con enfermedades sistémicas y pacientes portadores de prótesis parcial removible provisional tipo wipla.

Se empleó una matriz de datos que contiene las siguientes variables: genero, edad, arcada, clase de endetulismo parcial, estado gingival, estado de higiene oral, las variables retención, movilidad, recesión y defecto de furcación se valorizo en no presenta y presenta, estado de PPR valorizada en bueno (adaptado, sin fractura) y malo (desadaptado, fracturado) y estado periodontal valorizada en sano (estado gingival $=$ leve, estado de higiene $=$ adecuada, para las variables retención, movilidad, recesión y defecto de furcación = ausencia de estas características) y enfermo (estado gingival = moderado o severo, estado de higiene $=$ aceptable o deficiente, para las variables retención, movilidad, recesión y defecto de furcación $=$ presencia de estas características).

Para el análisis descriptivo univariado y bivariado utilizando las pruebas estadísticas de $\mathrm{t}$ de Student, Chi cuadrado de Pearson y prueba exacta de Fisher. Se tomó en cuenta un valor de $\mathrm{p}<0,05 \mathrm{y}$ el intervalo de confianza del $95 \%$ como el nivel de significancia estadística.

El protocolo de investigación fue revisado y aprobado por las autoridades de la Facultad de Estomatología, Departamento Académico de Clínica Estomatológica (DACE) para acceder a la información de las historias clínicas y por el Comité Institucional de Ética (CIE) de la Universidad Peruana Cayetano Heredia.

\section{RESULTADOS}

Se evaluaron 130 historias clínicas encontrando que el universo está conformada por el género femenino $(66.92 \%)$ y un $33.08 \%$ para el género masculino, con una edad media de 43.57 años, y el rango de mayor prevalencia fue entre 40 a 49 años (35.38\%).

Se realizó un análisis descriptivo univariado encontrando en la arcada inferior (61.54\%) y superior $(38.46 \%)$. Según la variable clase de edentulismo se encontró en la clase III $(57.69 \%)$, seguido de la
Clase II (27.69\%), luego de la Clase I (11.54\%), y finalmente la Clase IV (3.08\%). Para la variable estado gingival se encontró estado leve (55.38\%), moderado $(43.85 \%)$ y severo $(0.77 \%)$. La variable higiene oral se encontró estado deficiente (46.92\%), aceptable $(42.31 \%)$ y adecuado $(10.77 \%)$. La variable movilidad se encontró ausencia $(91.54 \%)$ y presencia $(8.46 \%)$. La variable retención se encontró presencia $(88.46 \%)$ y ausencia $(11.54 \%)$.

La variable recesión se encontró ausencia (79.23\%) y presencia (20.77\%). En cuanto al variable defecto de furcación, todo el universo carecía de este defecto. Por otro lado, la variable estado de PPR se encontró en buen estado (26.15\%) y mal estado (73.85\%). La variable estado periodontal se encontró periodonto sano (3.08\%) y periodonto enfermo (96.92\%). (Tabla 1)

Para las variables edad y sexo de paciente se encontró para el género femenino la edad media es de 42.76 años, donde 20 es la edad mínima y 64 la edad máxima; existiendo mayor prevalencia entre 40 a 49 años $(36.78 \%)$ y con respecto al género masculino la edad media es de 45.51 años, donde 27 años es la edad mínima y 64 es la edad máxima; existiendo mayor prevalencia entre 50 a 59 años (37.21\%).

También se realizó un análisis descriptivo bivariado donde el estudio no encontró relación estadísticamente significativa $(p>0.05)$ entre las siguientes variables:

- Estado periodontal - Género.

- Estado periodontal - Arcada dentaria.

- Estado gingival - Clase de edentulismo.

- Recesión gingival - Retención de placa.

- Estado de PPR - Estado gingival.

- Estado de PPR - Movilidad dentaria.

- Estado periodontal - Estado de PPR.

Sin embargo el estudio si encontró relación estadísticamente significativa $(p<0.05)$ entre las siguientes variables:

- Estado periodontal - Clase de edentulismo.

- Estado gingival - Higiene oral.

\section{DISCUSIÓN}

Con respecto al género, Awuapana y Col. (7), Hugo y col. (8) y Sánchez y Col. (9), encontraron 
Tabla 1. Variables de interés de los pacientes portadores de PPR, que acudieron a la Clínica Dental Docente de la UPCH, años 2011-2012.

\begin{tabular}{|c|c|c|c|}
\hline VARIABLE & CATEGORÍA & $\mathbf{N}$ & $\%$ \\
\hline \multirow{2}{*}{ Género } & Femenino & 87 & 66.92 \\
\hline & Masculino & 43 & 33.08 \\
\hline \multirow{5}{*}{ Rango de Edad } & 20 - 29 años & 16 & 12.33 \\
\hline & 30 - 39 años & 28 & 21.53 \\
\hline & 40 - 49 años & 46 & 35.38 \\
\hline & 50 - 59 años & 36 & 27.69 \\
\hline & 60 - 64 años & 4 & 3.07 \\
\hline \multirow{2}{*}{ Arcada } & Superior & 50 & 38.46 \\
\hline & Inferior & 80 & 61.54 \\
\hline \multirow{4}{*}{ Clase de Edentulismo } & Clase I & 15 & 11.54 \\
\hline & Clase II & 36 & 27.69 \\
\hline & Clase III & 75 & 57.69 \\
\hline & Clase IV & 4 & 3.08 \\
\hline \multirow{3}{*}{ Estado Gingival } & Leve & 72 & 55.38 \\
\hline & Moderada & 57 & 43.85 \\
\hline & Severa & 1 & 0.77 \\
\hline \multirow{3}{*}{ Higiene Oral } & Adecuada & 14 & 10.77 \\
\hline & Aceptable & 55 & 42.31 \\
\hline & Deficiente & 61 & 46.92 \\
\hline \multirow{2}{*}{ Movilidad } & No presenta & 119 & 91.54 \\
\hline & Presenta & 11 & 8.46 \\
\hline \multirow{2}{*}{ Retención } & No presenta & 15 & 11.54 \\
\hline & Presenta & 115 & 88.46 \\
\hline \multirow{2}{*}{ Recesión } & No presenta & 103 & 79.23 \\
\hline & Presenta & 27 & 20.77 \\
\hline \multirow{2}{*}{ Furcación } & No presenta & 130 & 100 \\
\hline & Presenta & 0 & 0 \\
\hline
\end{tabular}

mayor prevalencia en el género femenino que presentaba alguna clase de edentulismo parcial ya que son más propensas a la pérdida de dientes que los hombres debido a que tienen mayores necesidades odontológicas con respecto a la estética, experimentando mayores tratamientos invasivos que perjudicando el tejido dentario (8).

Con respecto a la arcada comprometida con la PPR, Belaunde y col. (10), López y col. (11) y Awuapana y col. (7), encontraron mayor prevalencia de la arcada inferior, donde existe mayor acumulación de restos alimenticios durante y después de su ingesta. Por otro lado resaltan que la arcada superior es la menos afectada, gracias a la función protectora de la lengua que genera una autolimpieza sobre las superficies dentarias superiores.

Con respecto a la prevalencia de la clase de edentulismo parcial, en el Perú Noborikawa en el 2001 (12), López en el 2009 (11) y Belaunde en el 2011 (10), encontraron mayor prevalencia en la Clase I, notando que los años pasan y los resultados son los mimos, debido que la población en ese intervalo de tiempo no toma conciencia ni siquiera interés de prevenir la perdida dentaria y por otro lado el profesional no busca estrategias para disminuirlas.

Sin embargo investigaciones realizados en Europa, Asia y Medio Oriente, se encuentra que la Clase más prevalentes es la Clase III, así como Sadig (13) y Dwari (14). Estas mencionan que sus resultados tienen relación con el nivel socioeconómico e intelectual de la población; si tomamos en cuenta que el Perú en los últimos años ha crecido socioeconómicamente siendo un país en vías de desarrollo podemos respaldar dicha relación.

Con respecto al estado gingival, la mayoría de estudios que relacionan el estado periodontal y el uso de PPR mencionan resultados generales sin especificar el origen, ni el grado de inflamación. Sin embargo Yeung y Col. (15) y Akaltran y Col. (16), especifican que los conectores mayores, placas proximales y brazos retentivos u opositores favorecen la retención y acumulación de placa blanda, los cuales invaden el tejido de soporte generando características desfavorables a nivel gingival. 
Los resultados sobre la higiene, revelan que los pacientes con PPR no tienen interés sobre los cuidados de su cavidad bucal, ni de su prótesis; o de repente si tienen interés pero no lo realizan adecuadamente, evidenciando características desfavorables en el periodonto.

Con respecto a la movilidad, Jim y Col. (18), Akaltran y col. (16) y Sánchez y Col. (19), mencionan que un mal diseño de la PPR afecta la distribución de las fuerzas sobre los dientes, favoreciendo así, la movilidad. Por otro lado, Jorge y Col. (20), revelaron que no existe dicha movilidad en PPR con diseño adecuado y controles de higiene oral.

Con respecto a la retención, una higiene deficiente con PPR, ya sea en bueno o mal estado, es factor de retención de alimentos sino tienen los cuidados adecuados. Por otro lado Lindhe, menciona que la formación de placa calcificada es favorecida por la ubicación de los conductos excretores de las glándulas salivales mayores, ya que el calcio y el fosfato de la saliva promueven su mineralización a largo plazo (1). Con respecto a la recesión, Sánchez y col. (19) y Cameron y col. (21), mencionan que los componentes de la PPR al estar en contacto íntimo con el margen gingival pueden provocar su migración. Por otro lado, Cameron y Col. dicen, para realizar el diseño compatible a salud periodontal, se debe tomar en cuenta las medidas de Herdenson, para no generar la reacción de los tejidos blandos (21). Sin embargo Mazurat y Mazurat (22), en una revisión sistemática concluyeron que hay insuficiente la evidencia clínica que muestre la asociación entre el diseño adecuado de PPR y el riesgo a desarrollar enfermedad periodontal. Según la Furcación, todo el universo estudiado carecía de este defecto que puede deberse a la mínima presencia de piezas multirradiculares comprometidas con la PPR, si es que no la perdieron previamente a su elaboración, ya que piezas con este defecto no brindan garantías necesarias para el éxito del tratamiento protésico.

En conclusión, es de vital importancia realizar controles rigurosos del estado periodontal antes, durante y después de cualquier tratamiento realizado en la cavidad bucal, para garantizar su éxito, funcionalidad, longevidad y por ende bienestar al paciente.

\section{CORRESPONDENCIA}

Gian Marco Gonzáles Pinedo

Jirón Quipaypampa N 155, 2da. Zona Tahuantinsuyo. Independencia, Lima-Perú.

Teléfono: (01) 526-1450 / 991739324

Correo electrónico: gian.gonzales@upch.pe gianny_08_10@hotmail.com

\section{REFERENCIAS BIBLIOGRÁFICAS}

1. Lindhe J, Karring T, Lang N. Clinical Periodontology and Implant Dentistry. 5 ed. Singapore: Blackwell Munksgaard; 2008.

2. Donayre F y col. Manual de procedimientos clínicos en periodontología. Lima: UPCH. Facultad de Estomatología; 1994.

3. Maetahara D. Prevalencia, extensión y severidad de recesiones gingivales en pacientes del hospital central de la fuerza aérea del Perú. Tesis para Título. Universidad Nacional Mayor de San Marcos; 2006.

4. Mallat E. Prótesis Parcial Removible. Madrid: Elsevier; 2004. p. 427-456.

5. Loza D. Prótesis Parcial Removible. Lima: Actualidades Médico Odontológicas Latinoamérica; 1992. p. 57.

6. Loza D, Valverde R. Diseño de Prótesis Parcial Removible. Madrid: Ripano; 2006. p. 13-19.

7. Awuapara N. Determinación de la asociación entre el edentulismo y la calidad de vida en la población adulta del distrito de Celendín, provincial de Celendín, departamento de Cajamarca en 2010. Tesis para Título. Universidad Peruana Cayetano Heredia; 2010.

8. Hugo F, Hilgert J, De Sousa M, da Silva D, Pucca J. Correlates of partial tooth loss and edentulism in the Brazilian elderly. Community Dent Oral Epidemiol. 2007;35:224-32.

9. Sánchez A. Estudio clínico longitudinal del efecto de las prótesis parciales removibles clínicamente validadas y el diseño empleado sobre la condición periodontal. Tesis para Título. Universidad de Venezuela; 2012.

10.Belaúnde M. Relación entre el acceso a la atención dental y el edentulismo en pobladores de 18 a 65 años de edad del distrito de Cajabamba, Provincia de Cajabamba, Departamento de Cajamarca, durante el periodo Febrero - Mayo del 2011. Tesis para Título. Universidad Peruana Cayetano Heredia; 2011.

11.López J. Prevalencia de edentulismo parcial según la Clasificación de Kennedy en el Servicio de Rehabilitación Oral del Centro Médico Naval "Cirujano Mayor Santiago Távara”. Tesis para Título. Universidad Nacional Mayor de San Marcos; 2009.

12.Noborikawa A. Frecuencia de pacientes edéntulos parciales según la clasificación de Kennedy - Applegate en la clínica universitaria de la Facultad de Odontología 
de la Universidad San Martín de Porres. Tesis para título. Universidad San Martín de Porres; 2001.

13.Sadig W. Removable Partial Denture Design: A study of a elected Population in Saudi Arabia. J Contemp Dent Pract. 2002 Nov 15;3(4):40-53.

14.Enoki K. Incidence of Partial Denture usage and Kennedy Classification. University of Osaka. Japan. Serie en internet. 2008 citado 3 Jul 2008; Disponible en: http://iadr.confex.com/iadr/2008toronto/techprogram/ abstract_106381.htm.

15. Yeung A, Lo E, Chow T, Clark R. Oral health status of patients 5-6 years after placement of cobalt- chromium removable partial dentures. J Oral Rehabil. 2000; 27(3):183-9.

16.Akaltan F, Kaynak D. An evaluation of the effects of two distal extension removable partial denture designs on tooth stabilization and periodontal health. J Oral Rehabil. 2005;32:823-9.

17.Carranza F, Sznajder N. Compendio de Periodoncia. 5 ed. Buenos Aires: Médica Panamericana; 1996. p. 21-25.
18.Jin $\mathrm{X}$, Sato $\mathrm{M}$, Nishiyama A, Ohyama T. Influence of loading positions of mandibular unilateral distal extension removable partial dentures on movements of abutment tooth and denture base. J Med Dent Sci. 2004;51(3):155-63.

19.Sánchez A. Estudio clínico longitudinal del efecto de las prótesis parciales removibles clínicamente validadas y el diseño empleado sobre la condición periodontal. Tesis para Título. Universidad de Venezuela; 2012.

20.Jorge J, Vergani C, Machado A, Pavarina A, Cardoso M. Clinical evaluation of abutment teeth of removable partial denture by means of the Periotestmethod. J Oral Rehabil. 2007;34(3):222-7.

21.Cameron S, Torres G, Lefler T, Parker H. The dimensions of mandibular lingual tisues relative to the placement of a lingual bar major connector. J Prosthodont. 2002;11:74-80.

22. Mazurat N, Mazurat R. Discus before fabricating: Communicating the realites of partial denture therapy. Part II: clinical outcomes. J Can Dent Assoc. 2003;69:96-100.

Recibido : 15-07-2013

Aceptado: 27-10-2013 